\title{
Tidal Stablization of Neutron Stars and White Dwarfs
}

\author{
Dong Lai \\ Theoretical Astrophysics, 130-33, California Institute of Technology \\ Pasadena, CA 91125 \\ E-mail: dong@tapir.caltech.edu \\ (Accepted for publication in Physical Review Letters, 1996)
}

\begin{abstract}
What happens to a neutron star or white dwarf near its maximum mass limit when it is brought into a close binary orbit with a companion? Such situation may occur in the progenitors of Type Ia supernovae and in coalescing neutron star binaries. Using an energy variational principle, we show that tidal field reduces the central density of the compact object, making it more stable against radial collapse. For a cold white dwarf, the tidal field increases the maximum stable mass only slightly, but can actually lower the maximum central density by as much as $30 \%$. Thus a white dwarf in a close binary may be more susceptible to general relativistic instability than the instability associated with electron capture and pycronuclear reaction (depending on the white dwarf composition). We analyse the radial stability of neutron star using post-Newtonian approximation with an ideal degenerate neutron gas equation of state. The tidal stablization effect implies that the neutron star in coalescing neutron star-neutron star or neutron star-black hole binaries does not collapse prior to merger or tidal disruption.
\end{abstract}

PACS Numbers: 97.80.Fk, 04.25.Dm, 04.40.Dg, 97.60.Jd

\section{Introduction}

It is well-established that there exist upper limits to the mass and central density of degenerate compact objects, white dwarf (WD) and neutron star (NS) [1.2]. For WD, the mass asymptotes to the Chandrasekhar limit as the central density increases, bounded by general relativistic radial instability, electron capture or pycronuclear reaction. For NS, the existence of an absolute mass limit is truly a general relativistic phenomena: pressure, which supports the star against gravity, also acts as a source of gravitation.

WD and NS frequently appear in binary systems. Type Ia supernova is thought to arise from accreting WD in a close binary or the merger of a WD-WD binary [3]. Of particular interest is the coalescing NS-NS binaries and NS-black hole $(\mathrm{BH})$ binaries, which are the most promising sources of gravitational waves that could be detected by interferometers such as LIGO and VIRGO [4 6 . NS binary merger is also considered to be a natural engine that drives cosmological gamma-ray bursts. Although the inspiral at larger orbital radius may be treated by post-Newtonian expansion technique [7], coupled with semi-analytic studies of the hydrodynamical effects [8] 13], quantitative understanding of the orbital evolution at small separation and the final merging requires full numerical simulation, which still is in its infancy 14 18].

The present study is motivated by the recent general relativistic hydrodynamical simulations of Wilson et al. [19] which reveal evidence that "general relativistic effects may cause otherwise stable neutron stars to individually collapse prior to merging". Obviously, this requires the neutron star to have a mass close to its maximum value to begin with (at large separation). However, it does raise a question as to how tidal field modifies the mass limit and central density limit of a compact object. In this paper, we analyse the radial stability of neutron star and white dwarf under the influence of the tidal field of a companion. We show that the tidal effect generally stablizes the star. We adopt the energy variational principle as widely used in the analysis of stability of isolated WD and NS [20, 1.2].

\section{General Consideration}

Let $E(\rho)$ be the energy of an isolated object of baryon mass $M$ as a function of the central density $\rho$. The function $E(\rho)$ consists of internal energy and self-gravitational energy, including their relativistic corrections. The equilibrium central density $\rho_{0}$ is obtained from the condition $(\partial E / \partial \rho)=0$; radial stability requires $\left(\partial^{2} E / \partial \rho^{2}\right)>0$.

Now for the star in a binary, the dimensionless tidal distortion is of order $\sim \varepsilon \equiv\left(M^{\prime} / M\right)(R / r)^{3}$, where $R \propto(M / \rho)^{1 / 3}$ is the mean stellar radius, $M^{\prime}$ the companion mass, $r$ the orbital separation. The total stellar energy can be written as $\mathcal{E}(\rho)=E(\rho)+W_{t}(\rho)$. Note that the tidal distortion modifies the "intrinsic" energy $E(\rho)$, but we will group this correction into $W_{t}(\rho)$. Thus the function $W_{t}(\rho)$ consists of (i) the correction $\Delta W$ to the self-gravitational potential energy, (ii) the interaction energy $W_{i}$ between $M^{\prime}$ and the tide-induced quadrupole, and (iii) the kinetic energy $T_{s}$ of internal fluid oscillation and rotation. The first two contributions are of the same order $\sim\left(G M^{2} / R\right) \varepsilon^{2} \propto 1 / r^{6}$, 
but have opposite signs, while the third $T_{s} \sim M R^{2} \varepsilon^{2} \Omega^{2} \propto 1 / r^{9}$ is a factor $\left(1+M^{\prime} / M\right)(R / r)^{3}$ smaller, and will be neglected [.

To calculate $W_{t}$, we model the star as a polytrope (with equation of state $P=K \rho^{1+1 / n} ; n$ is the polytropic index). Approximating the tidaly deformed star as an ellipsoid with axes $a_{i}=R\left(1+\alpha_{i}\right)$ ( $a_{1}$ is along the tidal bulge), the tidal distortion can be calculated in Newtonian theory. To leading order in $\varepsilon$, we have 21.

$$
\alpha_{1}=\frac{5}{2} q_{n} \frac{M^{\prime}}{M}\left(\frac{R}{r}\right)^{3}, \quad \alpha_{2}=\alpha_{3}=-\frac{5}{4} q_{n} \frac{M^{\prime}}{M}\left(\frac{R}{r}\right)^{3}
$$

where $q_{n}=\kappa_{n}(1-n / 5)$, and $\kappa_{n}$ is defined such that the moment of inertia of an isolated object is $I=(2 / 5) \kappa_{n} M R^{2}$ $\left(\kappa_{n}\right.$ specifies the mass concentration within the star). Note that $\alpha_{1}+\alpha_{2}+\alpha_{3}=0$ to leading order in $\varepsilon$. The two contributions to $W_{t}$ are

$$
\begin{aligned}
\Delta W= & \frac{3}{5-n} \frac{G M^{2}}{R} \frac{4}{45}\left(\alpha_{1}^{2}+\alpha_{2}^{2}+\alpha_{3}^{2}-\alpha_{1} \alpha_{2}-\alpha_{2} \alpha_{3}\right. \\
& \left.-\alpha_{1} \alpha_{3}\right)=\frac{3}{4} \kappa_{n} q_{n} \frac{G M^{\prime 2} R^{5}}{r^{6}},
\end{aligned}
$$

and

$$
\begin{aligned}
W_{i} & =-\frac{G M^{\prime}}{2 r^{3}}\left(\frac{2}{5} \kappa_{n} M R^{2}\right)\left(2 \alpha_{1}-\alpha_{2}-\alpha_{3}\right) \\
& =-\frac{3}{2} \kappa_{n} q_{n} \frac{G M^{\prime 2} R^{5}}{r^{6}} .
\end{aligned}
$$

Thus, up to order $\varepsilon^{2}$, the total tidal energy is

$$
W_{t}=-\lambda \frac{G M^{\prime 2} R^{5}}{r^{6}} \sim-\lambda \frac{G M^{\prime 2}}{r^{6}}(M / \rho)^{5 / 3},
$$

with $\lambda=(3 / 4) \kappa_{n} q_{n}$. Note that the negative sign in $W_{t}$ is crucial for the tidal stablization of radial mode.

The equilibrium condition in the presence of tide requires $\partial \mathcal{E} / \partial \rho=0$. Thus the density change $\delta \rho=\rho-\rho_{0}$ (where $\rho_{0}$ is the equilibrium density of the isolated object) due to the tidal field is given by

$$
\delta \rho=\left.\frac{5 W_{t}}{3 \rho}\left(\frac{\partial^{2} E}{\partial \rho^{2}}\right)^{-1}\right|_{\rho_{0}},
$$

with the expression evaluated at $\rho_{0}$. Since $\left(\partial^{2} E / \partial \rho^{2}\right)>0$ for stable configuration, we see that $\delta \rho<0$, i.e., the tidal field reduces the central density of a stable object. If the star is not too close to the stability limit, we have an estimate $\delta \rho / \rho_{0} \sim\left(-\varepsilon^{2}\right)$, which is of second order in the tidal deformation $\varepsilon$. Heuristically, a tidally-distorted object is less bound gravitationally (compared to a spherical object of the same mass), thus its volume expands in order to satisfy hydrostatic equilibrium.

Now consider how the tidal field changes the maximum mass $M_{m}$ of the object. Let $\rho_{m 0}$ and $M_{m 0}$ be the zerotide values of the maximum density and maximum mass, at which $\partial E / \partial \rho=\partial^{2} E / \partial \rho^{2}=0$ is satisfied. Taking the difference between $\left.(\partial \mathcal{E} / \partial \rho)\right|_{\rho_{m}, M_{m}}=0$ and $\left.(\partial E / \partial \rho)\right|_{\rho_{m 0}, M_{m 0}}=0$, we obtain

$$
\delta M_{m}=M_{m}-M_{m 0}=\left.\frac{5 W_{t}}{3 \rho}\left(\frac{\partial^{2} E}{\partial \rho \partial M}\right)^{-1}\right|_{\rho_{m 0}, M_{m 0}} .
$$

\footnotetext{
${ }^{1}$ We assume the star has negligible viscosity to be tidally synchronized, as is the case for compact objects 8.9. We also assume the star has zero intrinsic spin - Finite spin tends to stablize the star against radial collapse, and can be treated separately (e.g., 21 ). Note that there is no ambiguity in our definition of the function $\mathcal{E}(\rho)$ for stability analysis: One could add to $\mathcal{E}(\rho)$ a term $T=T_{s}+T_{\text {orb }}$ associated with the kinetic spin and orbital energies (so that $\mathcal{E}$ can be considered as the total energy of the binary system). However, differentiation of $T$ with respect to $\rho$ under fixed total angular momentum $J$ and fluid circulation $\mathcal{C}$ gives $(\partial T / \partial \rho)_{J, \mathcal{C}}=2 T_{s} / 3 \rho$, and the orbital motion does not affect the stellar structure.
} 
Since we can show $2\left(\partial^{2} E / \partial \rho \partial M\right)<0$, we find $\delta M_{m}>0$, i.e., the tidal field increases the maximum mass for stability. An order of magnitude estimate gives $\delta M_{m} / M_{m 0} \sim \lambda \varepsilon^{2}$.

Note that two implicit assumptions have been made in our analysis: (i) The star "relaxes" to its equilibrium shape even when the density is out of equilibrium. This separation of the radial motion and the "shape adjustment" makes our analysis of radial instability more transparent. It is valid because near the stability limit, the radial oscillation has almost zero frequency. (ii) The displacement of a fluid element inside the star is a linear function of the fluid position. This is exact only for the $n=3$ polytrope and in the incompressible limit, but otherwise corresponds to an approximate trial wavefunction in the variational principle.

\section{White Dwarf}

We now consider the stability of white dwarfs. Near the maximum mass, the stellar density profile resembles that of a $n=3$ polytrope. The "intrinsic" energy can be written as [1.2]

$$
E=E_{\text {int }}+W+\Delta E_{\text {int }}+\Delta E_{G R}
$$

where $E_{\text {int }} \sim M \rho^{1 / 3}$ is the internal energy of the ultra-relativistic electrons, $\Delta E_{\text {int }} \sim M \rho^{-1 / 3}$ is the correction due to finite electron mass, $W \sim-M^{2} / R \sim-M^{5 / 3} \rho^{1 / 3}$ is the potential energy of self-gravity, and $\Delta E_{G R} \sim-\left(M^{2} / R\right)(M / R) \sim-M^{7 / 3} \rho^{2 / 3}$ is the general relativistic (post-Newtonian) correction. For isolated cold $\mathrm{WD}$, this gives for the maximum central density and maximum mass $\rho_{m 0}=2.737 \times 10^{10} \mathrm{~g} \mathrm{~cm}^{-3}, M_{m 0}=1.4156 M_{\odot}$, the correcponding minimum radius $R_{m 0}=1110 \mathrm{~km}$ (We assume that the number of electron per nucleon is $Y_{e}=0.5$ ).

We parametrize the strength of the tidal field by the dimensionless ratio $\beta \equiv\left(M^{\prime} / M_{m 0}\right)\left(R_{m 0} / r\right)^{3}$. To avoid tidal disruption we require $\beta \lesssim 0.1$. With $\lambda \simeq 0.01$ (for $n=3$ polytrope) we then have

$$
W_{t} \simeq-0.01 \beta^{2} \frac{G M_{m 0}^{2}}{R_{m 0}}\left(\frac{M}{M_{m 0}}\right)^{5 / 3}\left(\frac{\rho}{\rho_{m 0}}\right)^{-5 / 3} .
$$

Expressing mass in the units of $M_{\odot}$, density in $10^{10} \mathrm{~g} \mathrm{~cm}^{-3}$, energy in $10^{51} \mathrm{erg}$, the total energy can be written as

$$
\begin{aligned}
\mathcal{E}= & 3.7129 M \rho^{1 / 3}-2.8895 M^{5 / 3} \rho^{1 / 3}+0.0457 M \rho^{-1 / 3} \\
& -0.0105 M^{7 / 3} \rho^{2 / 3}-0.14 \beta^{2}(M / \rho)^{5 / 3} .
\end{aligned}
$$

Solving $(\partial \mathcal{E} / \partial \rho)=\left(\partial^{2} \mathcal{E} / \partial \rho^{2}\right)=0$ yields the maximum density and maximum mass as a function of $\beta$. The result is shown in Figure 1. The increase in the maximum mass is rather small $(\lesssim 0.1 \%)$. However, the maximum density decrease can be rather substantial, reaching as much as $\sim 30 \%$. For small $\beta$, we have $\delta M_{m} / M_{m 0} \simeq 0.05 \beta^{2}$, and $\delta \rho_{m} / \rho_{m 0} \simeq-17 \beta^{2}$. Figure 1 also shows the central density of three constant-mass sequences with mass slightly below $M_{m 0}$. We see that as the binary separation decreases ( $\beta$ increases), the central densities also decrease, and always remain smaller than the maximum density $\rho_{m}(\beta)$ allowed for radial stability.

The fact that the maximum density $\rho_{m}(\beta)$ for stability decreases with decreasing $r$ may have some interesting astrophysical consequences. The maximum central density of a normal Carbon-Oxygen white dwarfs is set by the threshold of electron capture on ${ }^{16} \mathrm{O}$, at $\rho_{\text {cap }}=1.9 \times 10^{10} \mathrm{~g} \mathrm{~cm}^{-3}$ (the pycronuclear reaction between ${ }^{12} \mathrm{C}$ may set in at smaller density, of order $10^{10} \mathrm{~g} \mathrm{~cm}^{-3}$; but this increases as the ${ }^{12} \mathrm{C}$ abundance decreases [22]). From Fig. 1 we see that $\rho_{m}(\beta)$ can drop below $\rho_{\text {cap }}$, therefore GR effect becomes more important than neutronization. This implies that an accreting white dwarf in close binary can be more susceptible to collapsing to NS by general relativistic radial instability. Of course, the real situation may be complicated by the finite temperature due to the high accretion rate.

\section{Neutron Star}

The equation of state of nuclear matter is uncertain. For the purpose of illustration, we adopt EOS to be that of an ideal degenerate neutron gas and analyse the radial stability in the post-Newtonian approximation. Assuming the stellar density profile to be that of the $n=3 / 2$ polytrope, the "intrinsic" energy can still be written as in Eq. (7), except that $E_{\text {int }} \sim M \rho^{2 / 3}$ is the internal energy of nonrelativistic neutron, $\Delta E_{\text {int }} \sim-M \rho^{4 / 3}$ is the correction due to special relativistic effect. The maximum baryon mass of an isolated NS bassed on this model is $M_{m 0}=1.1108 M_{\odot}$,

\footnotetext{
${ }^{2}$ Consider a sequence of stellar models parametrized by the central density. Differentiating $\partial E / \partial \rho=0$ we obtain: $\left(\partial^{2} E / \partial \rho^{2}\right)+$ $\left(\partial^{2} E / \partial \rho \partial M\right)(d M / d \rho)=0$. The stable branch of the sequence satisfies $\left(\partial^{2} E / \partial \rho^{2}\right)>0$ and $d M / d \rho>0$, while the unstable branch satisfies $\left(\partial^{2} E / \partial \rho^{2}\right)<0$ and $d M / d \rho<0$. In both cases we have $\left(\partial^{2} E / \partial \rho \partial M\right)<0$.
} 
the maximum central density $\rho_{m 0}=7.415 \times 10^{15} \mathrm{~g} \mathrm{~cm}^{-3}$, and the corresponding minimum radius $R_{m 0}=10.48 \mathrm{~km}$. We again parametrize the tidal strength by the ratio $\beta \equiv\left(M^{\prime} / M_{m 0}\right)\left(R_{m 0} / r\right)^{3}$. The tidal energy can then be written in the form of Eq. (8) except that we use $\lambda \simeq 0.1$ appropriate for $n=3 / 2$ polytrope (The uncertainty can be readily absorbed into the definition of $\beta$ ). The GR correction to the tidal potential energy is of order $\left(M^{\prime} / r\right)$ smaller, and is neglected. Expressing mass in the units of $M_{\odot}$, density in $10^{15} \mathrm{~g} \mathrm{~cm}^{-3}$, and energy $10^{53} \mathrm{erg}$, the total energy function can be written as

$$
\begin{aligned}
\mathcal{E}= & 0.85168 M \rho^{2 / 3}-1.5968 M^{5 / 3} \rho^{1 / 3}-0.02887 M \rho^{4 / 3} \\
& -0.16774 M^{7 / 3} \rho^{2 / 3}-7.356 \beta^{2}(M / \rho)^{5 / 3} .
\end{aligned}
$$

Figure 2 shows the maximum density $\rho_{m}$ and maximum mass $M_{m}$ as a function of $\beta$. We see that, as in the white dwarf case, $\rho_{m}$ decreases and $M_{m}$ increases with increasing $\beta$. For small $\beta$ ( $\lesssim 0.15$ ), we have

$$
\begin{aligned}
\frac{\delta M_{m}}{M_{m 0}} & \simeq-\frac{15 W_{t}}{4\left(2 E_{i n}+W\right)}=0.33 \beta^{2}, \\
\frac{\delta \rho_{m}}{\rho_{m 0}} & \simeq-\frac{5 W_{t}\left(28 E_{i n}+15 W\right)}{2 W\left(2 E_{i n}+W\right)}=-2.7 \beta^{2} .
\end{aligned}
$$

Figure 2 also depicts the densities of constant-mass sequences with $M$ slightly below $M_{m 0}$. Again, we see that these densities $\rho(\beta)$ always remain below $\rho_{m}(\beta)$, i.e., these stars are stable against radial perturbation until the tidal limit $\beta \simeq 0.23$ is reached.

Another way to look at the tidal effect on the radial stability is to construct sequence of stellar models with varying central densities at a given fixed $\beta$. Figure 3 shows the $M-\rho$ curves of several such sequences. Only models in the $d M / d \rho>0$ branch are stable, and the maximum density $\rho_{m}$ and maximum mass $M_{m}$ are given by the values at the turning point $d M / d \rho=0$. One can readily see that the effects of the tidal field is to raise $M_{m}$ and lower $\rho_{m}$. For $\beta \gtrsim 0.23$, no stable configuration exists, and this corresponds to the tidal disruption limit.

\section{Discussions}

The above analysis demonstrates that tidal effect tends to stabilize neutron star and white dwarf against radial collapse, at least within the framework of post-Newtonian theory. Our method is accurate for white dwarfs, but only approximate for neutron stars. However, we think it is unlikely that higher order GR corrections or the use of more sophisticated nuclear equation of state [19] will change our qualitative results for neutron stars, although the precise numbers can certainly change.

In the cases of NS-NS binaries, one might still consider the possibility of neutron star collapse prior to merging when there is stable mass transfer from its lower mass companion. However, by the time mass transfer starts, the binary must already have encountered the orbital dynamical instability as a result of strong tidal interaction [10]. This tidal instability, enhanced by general relativistic effects [23] leads to rapid coalescence of the binary within a few orbits 13,24 .

I thank Kip Thorne for comments on an earlier version of the paper and helpful suggestions. This research is supported by the Richard C. Tolman Fellowship in theoretical astrophysics at Caltech, and NSF Grant AST-9417371 and NASA Grant NAG 5-2756.

[1] Ya. B. Zel'dovich and I. D. Novikov, Relativistic Astrophysics, Vol. 1 (Univ. of Chicago Press: Chicago, 1971).

[2] S. L. Shapiro and S. A. Teukolsky, Black Holes, White Dwarfs and Neutron Stars (John-Wiley \& Sons: New York, 1983).

[3] S. E. Woosley and T. A. Weaver, Ann. Rev. Astron. Astrophys., 24, 205 (1986); D. Branch, M. Livio, L. R. Yungelson, F. R. Boffi and E. Baron, Pub. Astro. Soc. Pacific, 107, 1019 (1995); see also I. Iben and A. V. Tutukov, Astrophys. J. Suppl., 54, 335 (1984).

[4] A. Abramovici, et al., Science, 256, 325 (1992).

[5] C. Cutler, et al., Phys. Rev. Lett., 70, 2984 (1993).

[6] K. S. Thorne, in "Proceedings of Snowmass 94 Summer Study on Particle and Nuclear Astrophys. and Cosmology", eds. E. W. Kolb and R. Peccei (World Scientific, Singapore, 1995). 
[7] L. Blanchet, T. Damour, B. R. Iyer, C. M. Will and A. G. Wiseman, Phys. Rev. Lett., 74, 3515 (1995).

[8] L. Bildsten and C. Cutler, Astrophys. J., 400, 175 (1992).

[9] C. S. Kochanek, Astrophys. J., 398, 234 (1992).

[10] D. Lai, F. A. Rasio and S. L. Shapiro, Astrophys. J., 420, 811 (1994).

[11] A. Reisenegger and P. Goldreich, Astrophys. J. 426, 688 (1994).

[12] D. Lai, Mon. Not. Royal Astro. Soc. 270, 611 (1994).

[13] D. Lai and S. L. Shapiro, Astrophys. J., 443, 705 (1995).

[14] K. Oohara and T. Nakamura, Prog. Theor. Phys., 83, 906 (1990); M. Shibata, T. Nakamura and K. Oohara, ibid., 88, 1079 (1992); ibid., 89, 809 (1993);

[15] F. A. Rasio and S. L. Shapiro, Astrophys. J., 401, 226 (1992).

[16] M. B. Davies, W. Benz, T. Piran and F. K. Thielemann, Astrophys. J., 431, 742 (1994).

[17] X. Zhuge, J. M. Centrella, S. L. W. Mcmillan, Phys. Rev. D. 50, 6247 (1994).

[18] M. Ruffert, H.-T. Janka and G. Schäfer, Astro. Astrophys., in press (1996).

[19] J. R. Wilson and G. J. Mathews, Phys. Rev. Lett. 75, 4161 (1995); J. R. Wilson, G. J. Mathews and P. Marronetti, Phys. Rev. D., submitted (1996).

[20] B. K. Harrison, K. S. Thorne, M. Wakano and J. A. Wheeler, "Gravitation Theory and Gravitational Collapse" (Univ. of Chicago Press, 1965).

[21] D. Lai, F. A. Rasio and S. L. Shapiro, Astrophys. J., 423, 344 (1994).

[22] See, e.g., M. Sahrling, Astro. \& Astrophys., 284, 484 (1994); M. Hernanz, J. Isern, R. Canel, J. Labay \& R. Mochkovitch, ApJ, 324, 331 (1988); S. Ogata, H. Iyetomi \& S. Ichimaru, ApJ, 372, 259 (1991).

[23] The precise location of the "inner-most stable orbit" due to GR is still uncertain. See L. E. Kidder, C. M. Will and A. G. Wiseman, Phys. Rev. D. 47, 3281 (1993); N. Wex and G. Schafer, Class. Quant. Gravity, 10, 2729 (1993). See also 19.

[24] D. Lai and A. G. Wiseman, Phys. Rev. D., submitted (1996).

FIG. 1. The maximum central density (heavy solid line) and maximum mass (dashed line) of white dwarf as a function of the tidal ratio $\beta \equiv\left(M^{\prime} / M_{m 0}\right)\left(R_{m 0} / r\right)^{3}$. The solid lines show the central densities of three sequences with mass slightly smaller than maximum mass of a isolated WD $M_{m 0}=1.4156 M_{\odot}$.

FIG. 2. The maximum central density (heavy solid line) and maximum mass (dashed line) of neutron star as a function of the tidal ratio $\beta \equiv\left(M^{\prime} / M_{m 0}\right)\left(R_{m 0} / r\right)^{3}$. The solid lines show the central densities of three sequences with mass slightly smaller than maximum mass of an isolated NS $M_{m 0}=1.1108 M_{\odot}$.

FIG. 3. The mass-central density curves of sequences of NS models with constant values of $\beta$. ( $\beta=0$ corresponds to isolated NS). 


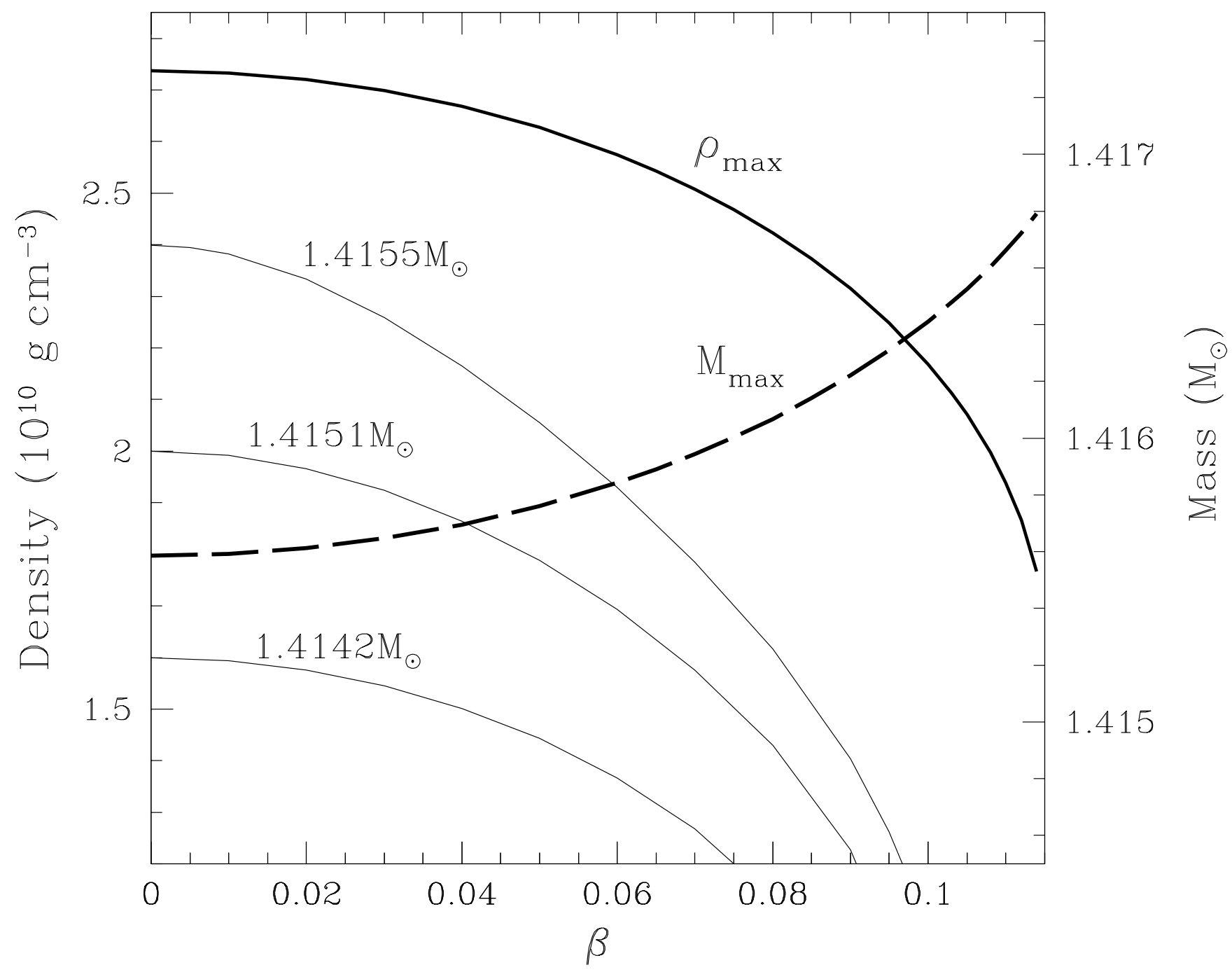

Fig. 1 


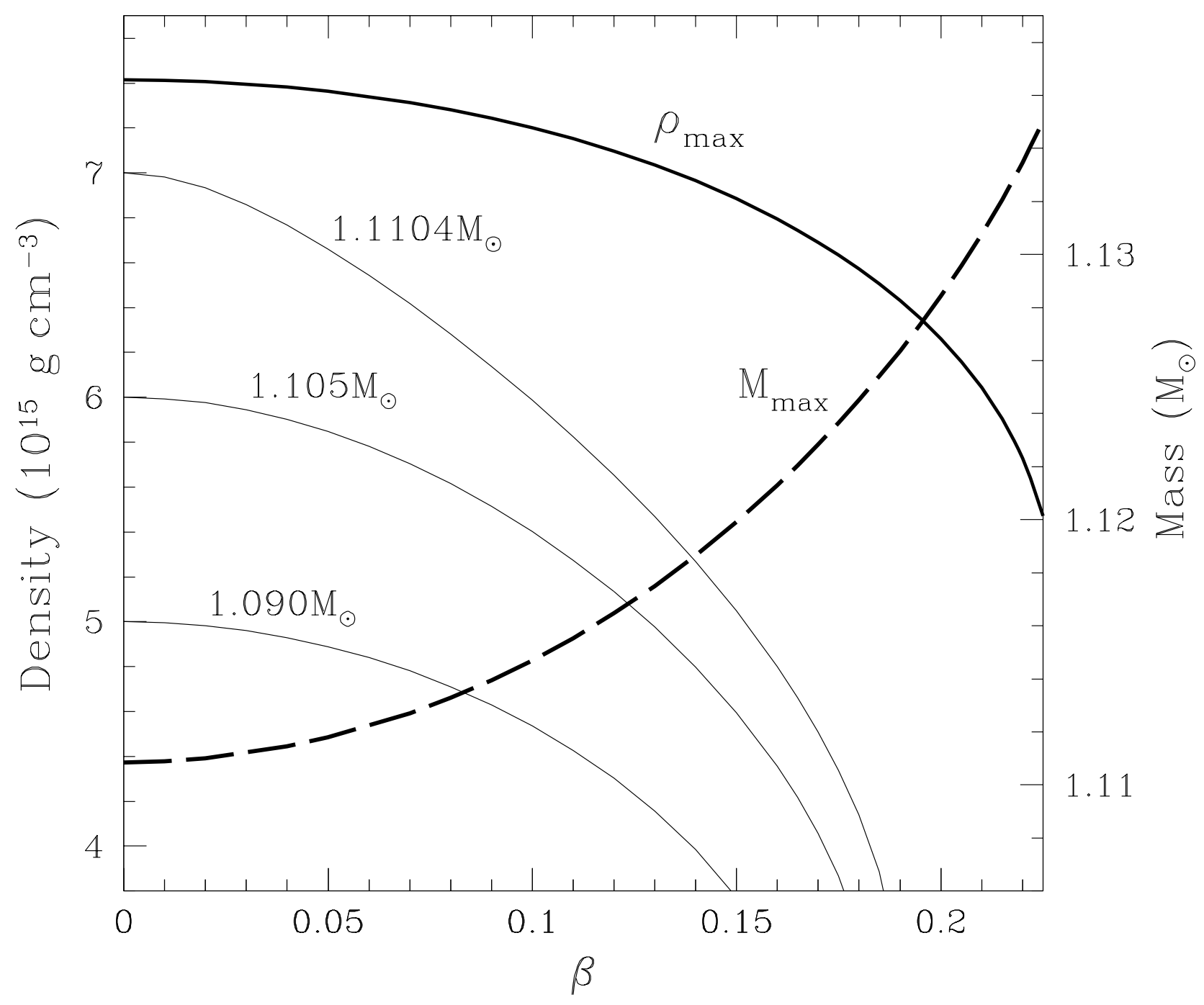

Fig. 2 


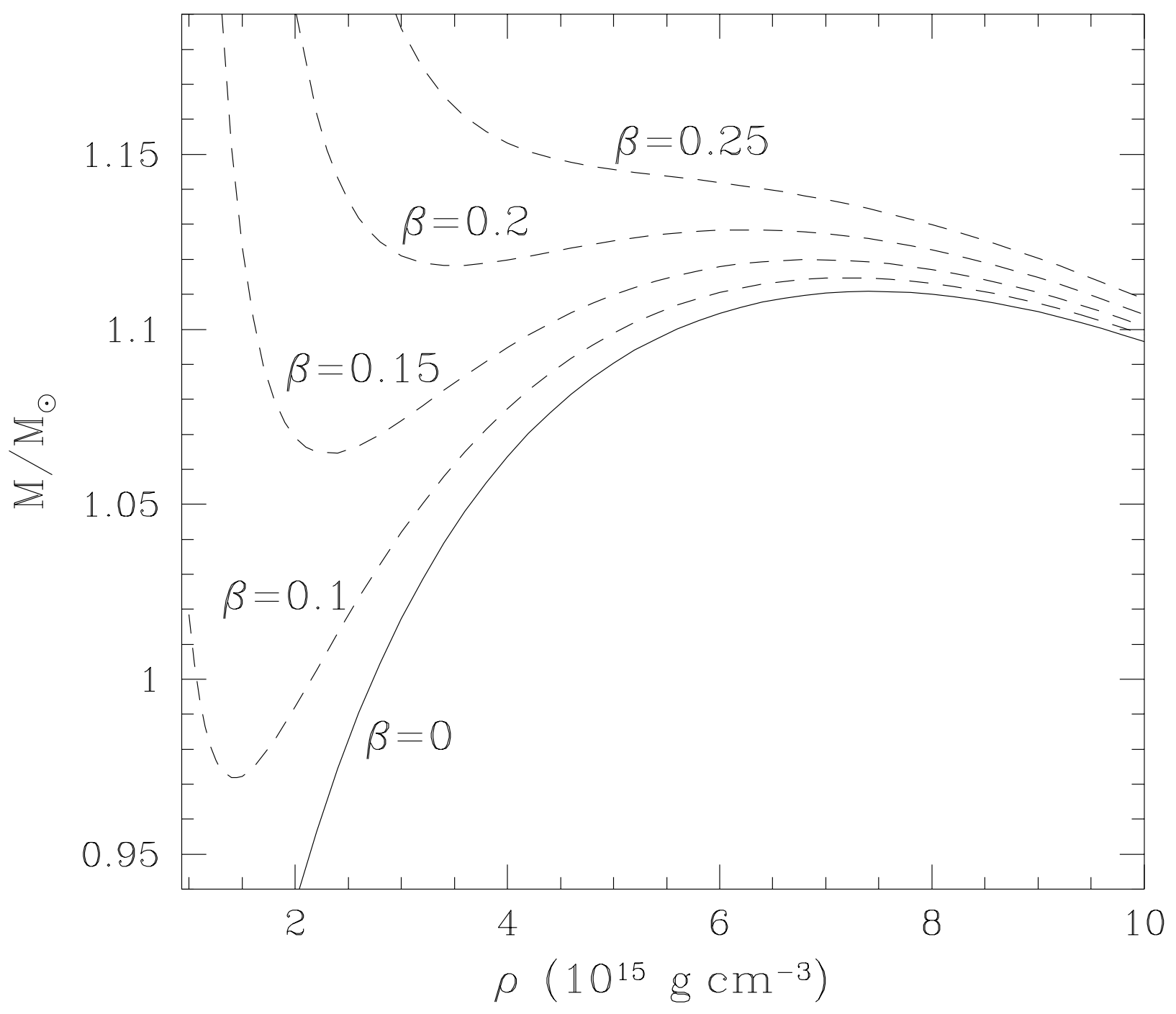

Fig. 3 